\title{
Acasalamento dirigido para aumentar a produção de animais geneticamente superiores e reduzir a variabilidade da progênie em bovinos
}

\author{
Haroldo Henrique de Rezende Neves ${ }^{1}$, Roberto Carvalheiro ${ }^{2,3}$, Vânia Cardoso ${ }^{2}$, Luiz Alberto \\ Fries $^{2 *}$, Sandra Aidar de Queiroz ${ }^{4}$
}

\footnotetext{
${ }^{1}$ Pós-graduação em Genética e Melhoramento Animal - FCAV/Unesp. Bolsista FAPESP

2 Gensys Consultores Associados S/C Ltda.

${ }^{3}$ Bolsista de Pós-Doutorado - FAPESP

${ }^{4}$ Departamento de Zootecnia - FCAV - UNESP - Jaboticabal. Bolsista do CNPq.

*In memoriam
}

RESUMO - Por meio da utilização de um programa de acasalamento dirigido e de simulação de dados, foram avaliadas e comparadas estratégias alternativas de acasalamento para aumentar a probabilidade de produzir animais superiores e reduzir a variabilidade da progênie em bovinos. Simularam-se 50 populações, cada uma com 4.800 vacas, 160 touros e 4.800 produtos. Para cada vaca e touro simulados, gerou-se uma variável aleatória, seguindo distribuição normal, reproduzindo um índice composto por diferenças esperadas na progênie (DEP) de diferentes características a ser adotado como critério de seleção. As estratégias de acasalamento simuladas foram: AA - ao acaso; AP - associativo positivo; AN - associativo negativo; C20 associativo positivo para as vacas $20 \%$ superiores e associativo negativo para as $80 \%$ restantes; C50 - associativo positivo para as vacas $50 \%$ superiores e associativo negativo para as $50 \%$ restantes. Os acasalamentos foram definidos com a utilização de um programa de acasalamentos dirigidos, calculando-se os índices esperados dos produtos e comparando as estratégias com base nestes índices. As estratégias de acasalamento alternativas, C20 e C50, apresentam probabilidades de produção de animais superiores tão altas quanto a do acasalamento associativo positivo e reduzem a probabilidade de produzir animais inferiores. $\mathrm{O}$ acasalamento associativo negativo é muito eficiente em produzir progênie uniforme.

Palavras-chave: associativo negativo, associativo positivo, DEP, índice, simulação

\section{Mating strategy for increasing the probability of producing outstanding animals and reducing progeny variability}

\begin{abstract}
A simulation study was carried out to apply and compare an alternative mating strategy for increasing the probability of producing outstanding animals and reducing progeny variability, with other conventional strategies. Fifty populations were simulated, each containing 4,800 dams, 160 sires and 4,800 calves. For each dam and sire, a random variable from a normal distribution was generated, mimicking an index composed by several expected progeny differences (EPD). The mating strategies considered were: (1) random mating (RM); (2) positive assortative mating (PA); (3) negative assortative mating (NA); (4) combined, PA (assortative mating) for $20 \%$ top dams, and NA (negative assortative mating) for the remaining $80 \%$ (C20); (5) combined, PA (positive assortative mating) for 50\% top dams, and NA (negative assortative mating) for the remaining 50\% (C50). After applying the mating program, the expected progeny indexes were computed and the strategies were compared based on these indexes. The alternative mating strategies, C20 and C50, show probability of producing outstanding animals as great as the positive assortative mating, and reduce the probability of producing extremely inferior animals. The negative assortative mating is very efficient in producing uniform progeny.
\end{abstract}

Key Words: EPD, index, negative assortative, positive assortative, simulation

\section{Introdução}

Estratégias de acasalamento dirigido permitem o uso mais racional dos animais geneticamente superiores de forma a alcançar os objetivos pré-estabelecidos em programas de melhoramento (Carvalheiro et al., 2007). Segundo Groen \& Van der Waaij (1999), o acasalamento dirigido pode ser feito com a combinação de touros e vacas semelhantes (acasalamento preferencial positivo - AP) ou com a combinação de touros e vacas não-similares 
(acasalamento preferencial negativo - AN), considerando o fenótipo ou genótipo dos animais como critério de semelhança, e o valor genético estimado (VGE) situa-se entre estes dois tipos de critério.

Considerando o efeito sobre a variabilidade na progênie, após uma única geração de acasalamento, parece haver uma dicotomia entre as estratégias de acasalamento associativo positivo e associativo negativo. $\mathrm{O}$ acasalamento associativo positivo aumenta a probabilidade de nascimento de animais com genótipos extremos superiores, o que, exceto para características com ótimo intermediário, é um resultado de grande interesse aos programas de melhoramento genético, que buscam melhores oportunidades de comercialização de material genético e a aceleração do progresso genético. Essa estratégia, contudo, também aumenta a probabili-dade de produção de animais extremos inferiores e a variabilidade da progênie, aumentando a proporção de fêmeas geneticamente inferiores para reposição e reduzindo a uniformidade entre os animais destinados ao abate. $\mathrm{O}$ acasalamento associativo negativo, no entanto, é indicado para situações de ótimo intermediário e/ou em que a uniformidade da progênie é desejada (Hohenboken, 1985; Carvalheiro et al., 2007).

Quinton \& Smith (1995), por meio de simulação estocástica, relataram que o acasalamento associativo positivo de animais selecionados com base na melhor predição linear não-viesada (BLUP) de seus valores genéticos proporcionou as maiores respostas quando ignorada a endogamia, mas apresentou menores respostas em comparação a outros métodos (AN e AA) no mesmo nível de endogamia.

Carvalheiro et al. (2007) também ressaltaram a possibilidade de combinação de acasalamento preferencial positivo e imposição de restrição sobre endogamia como meio de se alcançar maior progresso genético.

Realizou-se este trabalho com o objetivo de propor e avaliar estratégias alternativas de acasalamento dirigido por meio de estudo de simulação que permitissem, simultaneamente, aumentar a probabilidade de produzir animais geneticamente superiores e reduzir a variabilidade da progênie.

\section{Material e Métodos}

As estratégias de acasalamento estudadas foram comparadas por simulação dos dados. Foram simuladas 50 populações, cada uma composta por 4.800 vacas, 160 touros e 4.800 produtos (1 produto proveniente de cada vaca e 30 produtos de cada touro), semelhantemente à estrutura da população estudada por Cardoso et al. (2003). Cada população pode ser considerada equivalente a uma repetição para validação do processo estocástico de simulação.

Para cada vaca e touro simulados, gerou-se uma variável aleatória seguindo distribuição normal com média igual a 0 e desvio-padrão igual a 10. Essa variável hipotética pode ser interpretada, por exemplo, como um índice composto (IC) por DEP de diferentes características a ser adotado como critério de seleção e será assim referenciada posteriormente.

Os acasalamentos foram simulados conforme cinco estratégias, considerando-se os índices dos touros e vacas: AA - ao acaso; AP - associativo positivo; AN - associativo negativo; C20 - associativo positivo para as $20 \%$ melhores vacas e associativo negativo para as $80 \%$ restantes; C50 - associativo positivo para as $50 \%$ melhores vacas e associativo negativo para as $50 \%$ restantes.

Os acasalamentos associativo positivo, associativo negativo, C20 e C50 foram definidos com a aplicação do programa de acasalamentos dirigidos PAD (Roso \& Fries, 1998). Depois de definidos os acasalamentos, os índices esperados dos produtos foram calculados como a média aritmética dos índices de seus pais. As estratégias de acasalamento foram comparadas quanto à distribuição dos índices esperados dos produtos.

Também foram utilizados como critérios de comparação das estratégias de acasalamento os valores de média, variância, mínimo e máximo dos índices esperados dos produtos, a proporção de produtos com índice esperado acima de determinado ponto de truncamento e a média dos índices desses produtos.

O ponto de truncamento (t) adotado foi definido como a média mais um desvio-padrão do índice esperado dos produtos na situação de acasalamento ao acaso $(t=7,05)$. Todos os critérios de comparação foram calculados com base na média das 50 repetições simuladas.

\section{Resultados e Discussão}

As distribuições dos índices esperados dos produtos diferiram entre as estratégias (Figura 1). Para a estratégia de acasalamento associativo negativo, a maioria das observações concentrou-se próximo à média, ao contrário do que aconteceu no caso de acasalamento associativo positivo e ao acaso, com distribuições aproximadamente normais e maior variância para o acasalamento associativo positivo. Para as estratégias alternativas, as observações 
concentraram-se em torno de duas faixas de valores e com variância intermediária entre as variâncias de associativo positivo e ao acaso.

Conforme previsto, todas as estratégias de acasalamento simuladas apresentaram valor médio dos índices esperados dos produtos próximo a zero, embora a variância observada para cada estratégia tenha apresentado valores distintos (Tabela 1).

Em comparação ao acasalmento associativo negativo, o associativo positivo gerou índices esperados, com o dobro de variância, e aumentou em cerca de $50 \%$ o número de produtos que estariam acima do ponto de corte de 7,05 (Tabela 1).

Cardoso et al. (2003) discutiram as possíveis vantagens desse aumento, como o acréscimo da proporção de animais

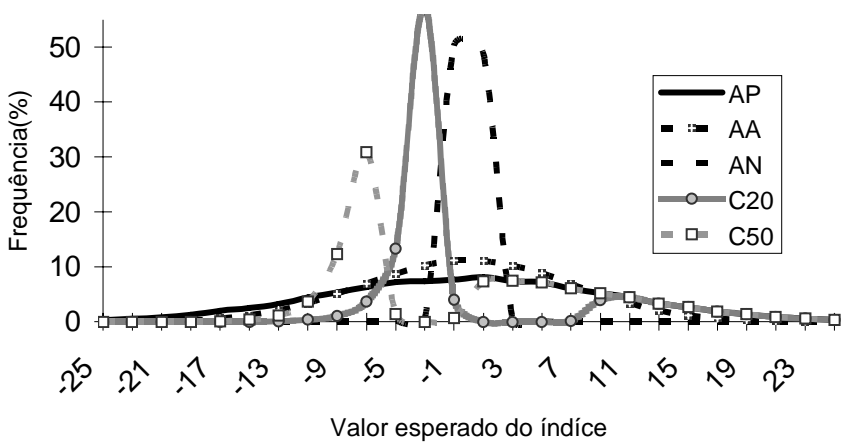

Figura 1 - Distribuição dos índices esperados dos produtos, conforme a estratégia de acasalamento adotada: ao acaso (AA); associativo positivo (AP); associativo negativo (AN); composto, AP para as vacas $20 \%$ superiores e AN para as $80 \%$ restantes (C20); composto, AP para as vacas $50 \%$ superiores e AN para as $50 \%$ restantes (C50).

Tabela 1 - Índices esperados dos produtos; proporção de produtos com índice esperado acima de 7,05 (I>7,05) e média dos índices desses produtos (média_top) em cada estratégia de acasalamento

\begin{tabular}{lrrrrr}
\hline & \multicolumn{5}{c}{ Estratégia de } \\
\cline { 2 - 6 } & Ao acaso & $\begin{array}{c}\text { Associativo } \\
\text { positivo }\end{array}$ & $\begin{array}{c}\text { Associativo } \\
\text { negativo }\end{array}$ & C20 & C50 \\
\hline Média & $-0,01$ & $-0,01$ & $-0,01$ & $-0,01$ & $-0,01$ \\
Variância & 49,71 & 99,02 & 0,37 & 54,48 & 82,53 \\
Mínimo & $-25,30$ & $-31,19$ & $-5,34$ & $-13,86$ & $-18,00$ \\
Máximo & 25,81 & 31,32 & 5,45 & 31,32 & 31,32 \\
I>7,05 (\%) & 16,03 & 24,12 & 0,01 & 20,00 & 24,12 \\
Média_top & 10,72 & 12,90 & 7,97 & 13,94 & 12,90 \\
\hline
\end{tabular}

C20 = composto: associativo positivo para as vacas $20 \%$ superiores e associativo negativo para as $80 \%$ restantes.

C50 = composto: associativo positivo para as vacas $50 \%$ superiores e associativo negativo para as $50 \%$ restantes. candidatos a receberem o certificado de superioridade genética (CEIP). No caso deste estudo, considerando DEP de nove características pré e pós-desmama para composição do índice de seleção, o uso do PAD permitiu aumento de $70 \%$ no número de produtos superiores e praticamente triplicou a variância dos produtos em relação à situação de acasalamento ao acaso.

A estratégia de acasalamento associativo positivo apresentou o menor valor de mínimo observado $(-31,19)$, que foi aproximadamente $23 \%$ menor que o observado na situação de acasalamento ao acaso, e esse resultado muitas vezes é um fator limitante para a implementação prática dessa estratégia de acasalamento. Cardoso et al. (2003) relataram redução de cerca de duas vezes no valor mínimo do índice esperado no caso da estratégia de acasalamento preferencial em relação à situação de acasalamento ao acaso, considerando DEP reais de nove características correlacionadas entre si e diferentes ponderações para composição do índice final.

$\mathrm{O}$ acasalamento associativo negativo foi eficiente em reduzir a variabilidade dos índices esperados da progênie (variância próxima a zero) e seria indicado para situações de ótimo intermediário e/ou em que maior uniformidade fosse desejada (Tabela 1). Essa estratégia, em muitos casos, já é usada empiricamente por alguns criadores, que procuram combinar touros e vacas com características diferentes e obtêm produtos mais uniformes entre si.

Ressalta-se que, para todas as estratégias de acasalamento, a variabilidade fenotípica da progênie dependeria ainda da variância de efeitos não-aditivos, de efeitos ambientais e da variância referente à segregação mendeliana, responsável por metade da variância genética aditiva.

De modo geral, as estratégias de acasalamento dirigido alternativas C20 e C50 atenderam o objetivo de aumentar a probabilidade de produzir animais geneticamente superiores e reduzir a variabilidade da progênie (Tabela 1 ; Figura 1). Os valores de máximo, a proporção de produtos com índice esperado acima de 7,05 e a média dos índices desses produtos para C20 e C50 foram iguais ou semelhantes aos valores observados no acasalamento associativo positivo.

Ainda em comparação ao acasalamento associativo positivo, os valores de mínimo observados para C20 e C50 foram 55 e $42 \%$ superiores, respectivamente, apesar de negativos. Embora os acasalamentos C20 e C50 tenham apresentado valores de variância média intermediários entre aqueles observados para os acasalamentos ao acaso e associativo positivo (Figura 1), grande parte (80 e 50\%, respectivamente) dos índices esperados gerados por C20 e 
C50 apresentaram variabilidade próxima àquela apresentada pelo acasalamento associativo negativo.

Uma possível consequência da busca por animais extremos, não estudada neste trabalho, seria o aumento do número de acasalamentos endogâmicos e, consequentemente do coeficiente médio de endogamia, o que poderia levar a piores respostas na estratégia de acasalamento associativo positivo em relação às de acasalamento associativo negativo e ao acaso (Quinton \& Smith, 1995). Entretanto, é possível controlar a endogamia ao direcionar os acasalamentos sem comprometer o mérito predito da progênie (Meuwissen, 1997; Kinghorn et al., 1999) e, inclusive, a combinação de acasalamento associativo positivo com restrição sobre endogamia poderia proporcionar maior progresso genético que o acasalamento aleatório (Carvalheiro et al., 2007).

Com este trabalho analisou-se o impacto de cada estratégia de acasalamento em uma única geração, mas outras simulações devem ser conduzidas para contrastar estratégias de acasalamento em situações de ciclos repetidos de seleção e acasalamento, com a simulação de diferentes gerações.

Outra estratégia interessante e que deve ser avaliada é a possibilidade de contemplar diversas estratégias de acasalamento alternativas associadas ao uso de sêmen sexado, visando atender diferentes condições de mercado, por exemplo, de modo a produzir touros, no caso dos produtos com índice mais elevado, e fêmeas de reposição, no caso dos produtos com índice na faixa de valores com maior freqüência de observações (Figura 1).

\section{Conclusões}

As estratégias alternativas de acasalamento são eficientes em elevar a proporção de animais geneticamente superiores e, ao mesmo tempo, reduzir a variabilidade da progênie, mediante a redução da proporção de animais inferiores. $\mathrm{O}$ acasalamento associativo negativo é muito eficiente em produzir progênie uniforme.

\section{Literatura Citada}

CARDOSO, V.; ROSO, V.M.; SEVERO, J.L.P. et al. Formando lotes uniformes de reprodutores múltiplos e usando-os em acasalamentos dirigidos, em populações Nelore. Revista Brasileira de Zootecnia, v.32, n.4, p.834-842, 2003.

CARVALHEIRO, R.; NEVES, H.H.R.; QUEIROZ, S.A. et al. Combinando acasalamento associativo positivo e restrição sobre a endogamia visando maior progresso genético. In: REUNIÃO ANUAL DA SOCIEDADE BRASILEIRA DE ZOOTECNIA, 44., 2007, Jaboticabal. Anais... Jaboticabal: Sociedade Brasileira de Zootecnia, [2007]. (CD-ROM).

GROEN, A.F.; WAAIJ, L.V.D. Some basics about mating schemes. In: INTERNATIONAL WORKSHOP EU CONCERTED ACTION ON GENETIC IMPROVEMENT OF FUNCTIONAL TRAITS IN CATTLE(GIFT): BREEDING GOALS AND SELECTION SCHEMES, 1999, Wageningen. Proceedings... Wageningen: Wageningen University and Research Center Publications, 1999. p.195-200.

HOHENBOKEN, W.D. The manipulation of variation in quantitative traits: a review of possible genetic strategies. Journal of Animal Science, v.60, p.101-110, 1985.

KINGHORN, B.P.; SHEPHERD, R.K.; WOOLLIAMS, J.A. An index of estimated breeding value, parental coancestry and progeny inbreeding to help maximize genetic gains. In.: ASSOCIATION FOR THE ADVANCEMENT OF ANIMAL BREEDING AND GENETICS, 1., 1999, Mandurah. Proceedings... Mandurah: 1999. v.13, p.412-415.

MEUWISSEN, T.H.E. Maximizing the response of selection with a predefined rate of inbreeding. Journal of Animal Science, v.75, p.934-940, 1997.

QUINTON, M.C.; SMITH, Comparison of evaluation-selection systems for maximizing genetic response at the same level of inbreeding. Journal of Animal Science, v.73, n.8, p.22082212, 1995.

ROSO, V.M.; FRIES, L.A. Um programa para planejar acasalamentos em bovinos de corte. In.: SIMPÓSIO NACIONAL DA SOCIEDADE BRASILEIRA DE MELHORAMENTO ANIMAL, 2., 1998, Uberaba. Anais... Uberaba: Sociedade Brasileira de Melhoramento Animal, 1998. p.359-360. 